Original article

\title{
Adverse drug reaction profile of anti-snake venom in a rural tertiary care teaching hospital
}

\author{
Rushikesh Prabhakar Deshpande*, Vijay Motiram Motghare, Sudhir Laxman Padwal, \\ Rakesh Ramkrishna Pore, Chetanraj Ghanshyam Bhamare, Vinod Shivaji Deshmukh, \\ Harshal Nutan Pise \\ Department of Pharmacology, Swami Ramanand Teerth Rural Government Medical College, Ambajogai, Beed, Maharashtra 431517, India
}

\section{A R T I C L E I N F O}

\section{Article history:}

Received 10 January 2013

Accepted 19 February 2013

Available online 2 April 2013

Keywords:

Snake bite

Envenoming

Antivenom

Antivenom reactions

Anaphylaxis

\begin{abstract}
A B S T R A C T
Snake bite is a major occupational hazard to farmers, labors especially in rural areas. Majority of the antivenom reactions are wrongly attributed to envenomation. The study was carried out with the aim of evaluation of the adverse drug reaction profile of anti-snake venom serum (ASV) in a rural tertiary care hospital. An observational study was conducted in SRTR Medical College, Ambajogai, Maharashtra, India. A total number of 296 indoor case papers of snake bite from February to September 2011 and June to August 2012 were retrieved from the record section and the antivenom reactions were assessed. In addition, basic epidemiological data and prescribing practices of ASV were also analyzed. Vasculotoxic snake bites were more common (50.61\%) than neuroparalytic ones (22.56\%). Mild envenomation was the commonest presentation. A total of 92 (56.10\%) patients who received ASV suffered from antivenom reactions. The most common nature of reaction was chills, rigors (69.56\%) followed by nausea and vomiting (34.8\%). 10-15\% patients suffered from moderate to severe reactions like hypotension and sudden respiratory arrest. We did not find any dose response relationship of ASV to risk of reactions (odds ratio 0.37). Intradermal sensitivity test was performed in about $72 \%$ cases. Our study showed a higher incidence of reactions to ASV at our institute.
\end{abstract}

Copyright (c) 2013, InPharm Association, Published by Reed Elsevier India Pvt. Ltd. All rights reserved.
The poison of snake and newt; Is sweat of "Envy's foot"

-William Blake (Auguries of Innocence).

\section{Introduction}

Snake bite is a common, neglected and frequently devastating environmental and occupational disease, especially in rural areas of tropical developing countries. It is a major public health problem in India with estimated annual snake bite incidence of about 250,000 out of which approximately $20 \%$ bites result in significant envenoming which require anti-snake venom administration. ${ }^{1}$ The snake bite associated morbidity is estimated to be about 1.4-68/ 1 lakh population, mortality about 1.1-2.4/1 lakh and case fatality rate of $1.7-20 \%{ }^{2}$ It is estimated that between $35,000-50,000$ people die of snake bite in India each year. ${ }^{3}$ Early in 2009, snake bite was included in the WHO's list of neglected tropical diseases. ${ }^{4}$

\footnotetext{
* Corresponding author. Tel.: +91 (0) 7709030305.

E-mail address: drrushikesh@gmail.com (R.P. Deshpande).
}

Anti-snake venom (ASV) and its rational use is the only definitive treatment to neutralize venom in circulation and in tissue fluid to save life in snake bite cases. Antivenom is an immunoglobulin usually pepsin refined $\mathrm{F}(\mathrm{ab})_{2}$ fragments of IgG purified from the serum or the plasma of a horse or sheep that has been immunized with the venom of one or more species of snakes. In India only polyvalent ASV is available. The antivenins are produced against 4 most important venomous snakes of India - Naja naja (Indian cobra); Bungarus caeruleus (Indian common krait); Daboi russelli (Russell's viper) and Echis carinatus (Saw-scaled viper). Each ml of polyvalent ASV produced in India neutralizes $0.6 \mathrm{mg}$ dried Indian cobra venom, $0.45 \mathrm{mg}$ dried common krait venom, $0.6 \mathrm{mg}$ of dried Russell's viper venom and $0.45 \mathrm{mg}$ of dried Saw-scaled viper venom. 5,6

Usually more than $20 \%$ cases develop either early (within few hours) or late (5 days or more) allergic reactions following ASV administration. $^{3}$ The deaths due to ASV reactions are wrongly attributed to envenomation. In addition, the significant problem of acute adverse reactions to ASV is compounded by a lack of appropriate recommendations regarding prevention, diagnosis and management of such reactions. ${ }^{7}$ 
Considering the prevalence of snake bites and severity of the problem at rural setup the present study was planned to evaluate the adverse drug reaction profile of ASV in a rural tertiary care teaching hospital.

\section{Subjects and methods}

\subsection{Ethics}

An observational study was conducted in Pharmacovigilance Centre at Swami Ramanand Teerth Rural Govt. Medical College, Ambajogai, Maharashtra, India. The ethical approval was obtained from Institutional Ethics Committee prior to initiation of the study (SRTRMC/PHARMAC/IEC/40B/2011).

\subsection{Patients}

Records of total number of 296 indoor cases admitted from February to September 2011 and June to August 2012 were retrieved from the medical record section of the institute and data was collected on a prevalidated case record proforma. Out of which, 164 cases in whom ASV was administered due to systemic envenomation were included in the study results. The first section of the data included demographic data like initials of patient, age, gender, weight, occupation and address. The second section was regarding the documentation of snake bite like type of snake bite, initial clinical presentation of the patient, intradermal sensitivity test done or not, 20 min whole blood clotting test (WBCT), dose, route and frequency of administration of ASV.

\subsection{Signs of adverse reactions}

The antivenom reactions were documented and classified as early anaphylactic, endotoxic (pyrogenic) and late (serum sickness type) reactions. The management of antivenom reactions was also recorded. The data was filled on suspected adverse drug reaction reporting form by Central Drug Standard Control Organization. The reactions were assessed by seriousness (death, life threatening, hospitalization-initial or prolonged, disability and required intervention to prevent permanent damage/impairment) and outcome (fatal, continuing, recovered and recovering). The outcome of the cases was analyzed under headings of recovery, referral and mortality.

\section{Results}

\subsection{Patient demographics}

Out of the 164 patients who received antivenom, 91 (55.49\%) were male and 73 (44.51\%) female. This can be attributed to the out-dwelling lifestyle and occupational outdoor stay of males. Incidence of snake bite was most common in the age group of 2130 years (27.36\%), followed by $31-40$ years and $11-20$ years (20.27\%). The majority of patients were farmers, plantation workers and laborers (Table 1 ). The incidence of antivenom reactions was highest in the age group of 21-40 years (Table 2). We found the incidence of vasculotoxic snake bites was more common (50.61\%) than neuroparalytic snake bites (22.56\%).

\subsection{Antivenoms used, dosage forms and routes of administration}

The anti-snake venom used at our setup during the study duration was manufactured by, Haffkine Biopharmaceutical Company Ltd. Mumbai, VINS Bioproduct Ltd. Hyderabad and Bharat Serum and Vaccines Ltd. Mumbai. The lyophilized antivenom was
Table 1

Demographic details of patients admitted with snake bite.

\begin{tabular}{lc}
\hline Age (in years) & No. of patients \\
\hline $0-10$ & 6 \\
$11-20$ & 60 \\
$21-30$ & 81 \\
$31-40$ & 60 \\
$41-50$ & 42 \\
$51-60$ & 24 \\
$>60$ & 23 \\
Sex & \\
Male & 164 \\
Female & 132 \\
Total & 296 \\
\hline
\end{tabular}

reconstituted with $10 \mathrm{ml}$ water to $10 \mathrm{ml}$ of ASV. The dose of ASV largely depends upon the signs of systemic envenomation and severity of bite. In our setup the dose of ASV used ranges from $50 \mathrm{cc}$ to $200 \mathrm{cc}$ depending upon signs and symptoms of the patient. The most commonly used loading dose of antivenom was $100 \mathrm{ml}$. The ASV was diluted and infused intravenously at a constant rate over a period of about one hour (Fig. 3).

\subsection{Intradermal skin testing to predict antivenom reaction prior to administration of ASV?}

Skin/conjunctival hypersensitivity testing does not reliably predict early or late antivenom reactions as they are mediated by direct activation of complement system and not mediated by $\operatorname{IgE}$ and is not recommended. ${ }^{3,8-10}$ However in our study, we found that in about $72 \%$ of cases the skin hypersensitivity test was performed prior to initiation of ASV therapy. Skin testing only delays the administration of ASV and can themselves be sensitizing. . $^{3,8,10}$

\subsection{Adverse antivenom reaction rates}

A total of 92 patients (56.10\%) who received ASV suffered from antivenom reactions. The most common presentation of reaction were chills, rigors (69.3\%) followed by nausea and vomiting (41.3\%). $10-15 \%$ patients suffered from moderate to severe reactions like hypotension and sudden respiratory arrest (Fig. 1 ). The literature by WHO states that the antivenom reactions are dose related, however in our study we did not find any statistically significant dose response relationship of ASV to risk of reactions (odds ratio 0.37) (Fig. 2). Some investigators believe that ASV reactions are seen more in hemotoxic bites than neurotoxic ones. In our study, there was a higher incidence of antivenom reactions in cases of vasculotoxic snake bites (52.17\%) as compared to neuroparalytic snake bites (21.74\%) (Table 3$)$.

Table 2

Age and gender wise distribution of antivenom reactions.

\begin{tabular}{|c|c|c|c|c|c|c|}
\hline \multirow[t]{2}{*}{ Age (years) } & \multirow[t]{2}{*}{ Gender } & \multicolumn{2}{|c|}{ Reaction present } & \multicolumn{2}{|c|}{ Reaction absent } & \multirow[t]{2}{*}{ Total } \\
\hline & & Number & $\%$ & Number & $\%$ & \\
\hline \multirow[t]{2}{*}{$\leq 20$} & Male & 14 & 15.22 & 12 & 16.67 & 45 \\
\hline & Female & 11 & 11.96 & 8 & 11.11 & \\
\hline \multirow[t]{2}{*}{$21-40$} & Male & 17 & 18.48 & 18 & 25.00 & 70 \\
\hline & Female & 23 & 25.00 & 12 & 16.67 & \\
\hline \multirow[t]{2}{*}{$41-60$} & Male & 6 & 6.52 & 14 & 19.44 & 36 \\
\hline & Female & 13 & 14.13 & 3 & 4.17 & \\
\hline \multirow[t]{2}{*}{$>60$} & Male & 5 & 5.43 & 5 & 6.94 & 13 \\
\hline & Female & 3 & 3.26 & 0 & 0 & \\
\hline Total & & 92 & & 72 & & 164 \\
\hline
\end{tabular}




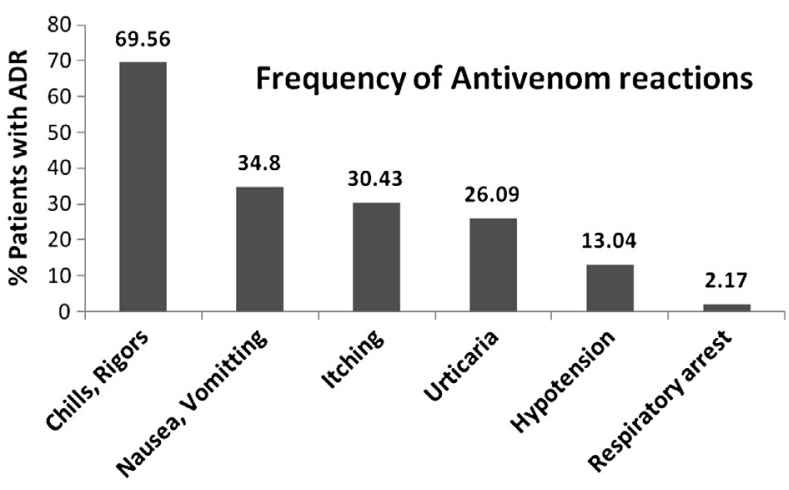

Fig. 1. Frequency of antivenom reactions.

\subsection{Characteristic features of snake bites}

Mild envenomation was the commonest presentation. The commonest symptoms of presentation was swelling at site of bite in case of vasculotoxic bite and drooping of eyelid in case of neuroparalytic snake bite. The commonest site of bite was found to upper limb in case of female and lower limb in case of male. The average journey time to hospital (AJTH) was $3 \mathrm{~h}$. The mean duration of hospitalization was 3 days; with outcome of more than $89 \%$ patients was recovered.

\section{Discussion}

Snake bite is a common occupational hazard of farmers, plantation workers who are generally from low socio-economic status, resulting in tens of thousands of deaths each year and many cases of chronic physical handicap.

\subsection{Role of antivenom}

The only specific antidote to the toxins in snake venom is hyperimmune globulin from an animal that has been immunised with the appropriate venom. ${ }^{11}$ The introduction of serum antivenom by Albert Calmette in 1895 for the treatment of envenoming was quickly accepted without formal clinical trials. ${ }^{12}$ More than a century later, antivenoms are considered as essential drugs.

ASV is prepared from horse serum and is associated with allergic reactions which may result in anaphylaxis and even death. It is costly and there is a constant shortage of ASV, particularly in rural and underdeveloped parts of India. Thus ASV should be used only when there is possibility of circulating venom in the body and not to all snake bite cases. However, early administration of ASV is essential to neutralize the maximum circulating venom before it is

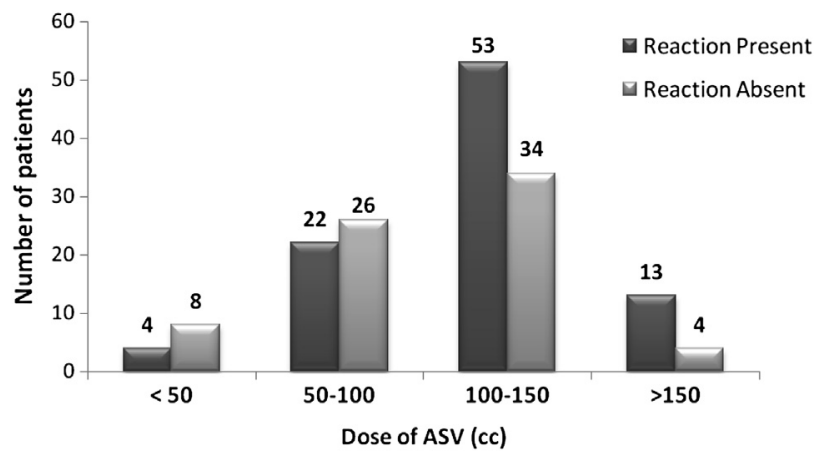

Fig. 2. Dose response to ASV in terms of ADR.

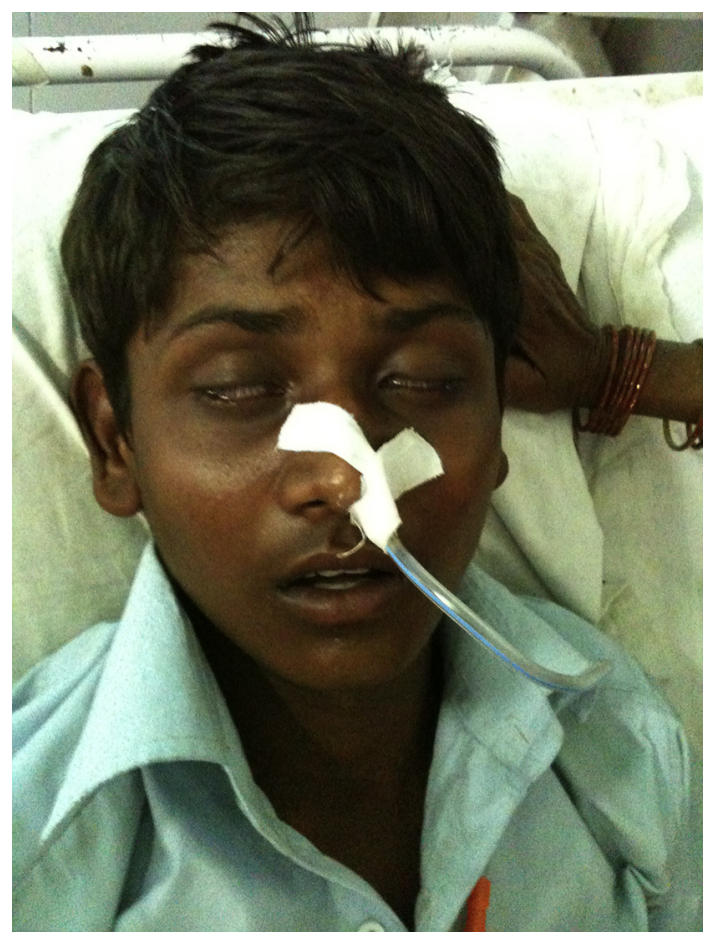

Fig. 3. Ptosis in neuroparalytic snake bite.

fixed in the tissue. Therefore, it should be given to cases with evidence of systemic envenomation as early as possible. Development of enlarged tender lymph node draining the bitten limb is an early manifestation of poisonous snake bite needing ASV therapy.

\subsection{Adverse reactions to antivenom and its prevention and treatment}

\subsubsection{Early anaphylactic reactions}

It usually occurs in 10-180 min of starting antivenom. It includes urticaria, itching (often over the scalp), cough, nausea, vomiting, abdominal colic, diarrhea and tachycardia. Minority of cases present with fatal anaphylaxis-hypotension, bronchospasm and angioneuritic edema. They occur due to direct activation of complement by IgG and residual FC fragment or direct stimulation of mast cells and basophils by antivenin proteins. They are not IgE mediated, type I reactions.

\subsubsection{Pyrogenic reactions}

It usually develops 1-2 h after starting ASV therapy. Fever, rigors, chills, hypotension are the presenting features. They are due to pyrogenic contamination of ASV and diluting fluid.

\subsubsection{Late serum sickness type reactions}

It develops in 1-12 days after antivenin therapy (mean 7 days). Clinical features include fever, nausea, arthralgia, myalgia, arthritis, mononeuritic multiplex, recurrent urticaria, lymphadenopathy,

Table 3

Reaction profile according to types of snake bite.

\begin{tabular}{llllll}
\hline Type of snake bite & \multicolumn{3}{l}{ Reaction to anti-snake venom } \\
\cline { 2 - 3 } & \multicolumn{2}{l}{ Present } & & Absent & \\
\cline { 2 - 3 } \cline { 5 - 6 } & Number & $\%$ & & Number & $\%$ \\
\hline Vasculotoxic & 48 & 52.17 & & 35 & 48.61 \\
Neuroparalytic & 20 & 21.74 & & 17 & 23.61 \\
Not specified & 24 & 26.09 & & 20 & 27.78 \\
Total & 92 & & & 72 & \\
\hline
\end{tabular}


neuritis and even encephalopathy. They usually respond to oral antihistamine.

Our study showed a higher incidence of reaction to ASV as compared to WHO literature, most of which were of early anaphylactic type or pyrogenic (endotoxic) in nature. Majority of the reactions were classified as "not preventable" by Schumock Thornton criteria. They were "expected", "hypersensitivity reactions" as per Karch and Lasanga classification of adverse drug reactions. With the use of modified Hartwig Siegel scale, majority of the antivenom reactions were mild and moderate in nature. The antivenom reactions were treated with adrenaline, antihistaminics and steroids. There was a trend of using prophylactic anti-histaminics and corticosteroids in anticipation of reactions. Attempts to prevent early reactions which included pretreatment with epinephrine, anti-histaminics, corticosteroids and reduction in speed and concentration of intravenous antivenom administration have not been effective in adequately designed clinical trials. ${ }^{13,14,15}$ However the studies by Premawardhena et al, ${ }^{16}$ Gawarammana et $\mathrm{al}^{17}$ and de Silve et $\mathrm{al}^{18}$ have shown the efficacy of low dose adrenaline and combination of chlorpheniramine and hydrocortisone in reduction of antivenom reactions. A recent systematic review and meta-analysis ${ }^{19}$ has shown the beneficial role of premedication with adrenaline in reduction of early antivenom reactions. Epinephrine is the most promising treatment for anaphylactic reactions, acts by reducing the capillary permeability and bronchospasm. However, the risks of epinephrine make it less attractive as a prophylactic treatment. ${ }^{20,21}$ In presence of such controversial data, the WHO has not recommended routine use of prophylactic drugs to prevent ASV induced reactions. In the absence of any proven effective method of prevention of antivenom reactions, patients should be observed carefully for at least $2 \mathrm{~h}$ after they are given antivenom, and epinephrine should be given at the first sign of anaphylaxis. Also, there was a poor documentation of adverse drug reactions to ASV in terms of onset, duration, outcome and seriousness.

\subsubsection{0 min whole blood clotting test (20 WBCT)}

This very useful and informative bedside test requires very little skill and one apparatus-clean, dry glass vessel. If the blood is still liquid (un-clotted) after $20 \mathrm{~min}$ and runs out, the patient has hypofibrinogenemia as a result of venom induced consumption coagulopathy. This test is important parameter for initiation and repetition of ASV in snake bite cases. In India whole blood clotting time of more than 20 min is virtually diagnostic of viper bite and rules out elapid bite. However our study has shown that in about $36 \%$ cases of vasculotoxic snake bites, 20 min WBCT was not done and it was done only in $28 \%$ cases of undiagnosed snake bites, indicating a poor management protocol. The clinical findings like prognostic signs, symptoms, type of snake bite and results of various investigations were not adequately documented in majority of case papers. Majority of the case records lacked documentation of history of atopic diseases and previous allergic reactions to any drugs or biological serum like anti rabies serum. There was no documentation of batch number or expiry date in any of the case paper studied.

We sent the samples of ASV at our institute during study period to the Central Drug Laboratory at Central Research Institute, Kasauli, Himachal Pradesh, India for quality check. The report (CDL/ 2011/6291 Dt. 05-10-11) has shown that all the samples of ASV were of standard quality in respect to potency, physical aspects, sterility, abnormal toxicity and biochemical tests. Thus we can rule out the faulty ASV as a cause of increased antivenom reactions in our study.

Snakebite injuries occur most frequently in rural areas of tropical developing countries where access to and quality of health care services create difficulties both for the prompt administration of antivenom and for the treatment of antivenom-related reactions. ${ }^{22}$ However, as our institute is a rural area based tertiary care hospital, the mean average journey time to hospital is less than $3 \mathrm{~h}$, ranging from $30 \mathrm{~min}$ to $24 \mathrm{~h}$, which made it possible to manage the cases of snake bites early. The outcome of snake bite cases is more favorable owing to the continuous and adequate supply of ASV and trained staff.

In conclusion, the management practices of snake bite cases and adverse drug reactions following ASV administration are far from satisfactory in our institute. There is a need of adequate documentation of adverse reactions in terms of onset, duration, severity and outcome. Also, there is underreporting of antivenom reactions of ASV to the Pharmacovigilance Centre at our institute. It is partly due to absence of efficient ADR monitoring system and partly because of lack of reporting culture among the treating physicians. It is a need of hour for Pharmacovigilance training in the form of CMEs or Workshops to the treating physicians for better diagnosis and reporting of adverse drug reactions.

This study provides insight into current clinical management of snake bite in rural health centers and provides incidence and severity of antivenom reactions. We plan to use the results of this study to conduct a prospective trial for antivenom premedication strategies in India.

\section{Conflicts of interest}

All authors have none to declare.

\section{References}

1. Warell DA, ed. WHO/SEARO Guidelines for the Clinical Management of Snake Bite in South East Asia Region. SE Asian J Trop Med Pub Health. 1999;30:1-85.

2. Chippaux JP. Snakebite: appraisal of global situation. Bull World Health Organ. 1998;76:515-524.

3. Warrell DA, ed. WHO/SEARO Guidelines for the Clinical Management of Snake Bite in South East Asia Region New Delhi; 2010:1-67.

4. WHO. Model list of essential medicinesAvailable from, http://www.int/ selection_medicines/committees/expert/17/sixteenth_adult_list_en.pdf; March 2009.

5. Deoras PJ. Snake in India. India: National Book Trust; 1965:33.

6. Pe Tun, Cho Khin Aung. Amount of venom injected by Russell's viper (Vipera russelli). Toxicon. 1988;24:730-733.

7. Simpson ID, Norris RL. Snake antivenom product guidelines in India: "The devil is in the details". Wilderness and Environ Med. 2007;18:163-168.

8. Warrell DA. Venomous and poisonous animals in mansion's tropical disease. In: Cook GC, et al., eds. 22nd ed. Saunders: Elsevier; 2009:557-581.

9. Malasit P, Warrell DA, Chanthavanich P. Prediction, prevention and mechanism in early (anaphylactic) antivenom reactions in victims of snakebite. BMJ (Clin Res Ed). 1986;292:17-20.

10. Cupo P, Azevedo-Marques MM, de Menezes JB, Hering SE. Immediate hypersensitive reaction after intravenous use of antivenom sera: prognostic value of intradermal sensitive test. Rev Inst Med Trop Sao Paulo. 1991;33:115-122.

11. WHO. Guidelines for the Production, Control and Regulation of Snake Antivenom Immunoglobulins. Geneva: World Health Organization; 2010.

12. Bon C. Serum therapy was discovered 100 years ago. In: Bon C, Goyffon M, eds. Envenoming and Their Treatments. Lyon: Fondation Marcel Merieux; 1996:3-9.

13. Warrell DA. Snake bite. The Lancet. 2010;375:77-88.

14. Fan HW, Marcopito LF, Cardoso JL, et al. Sequential randomised and double blind trial of promethazine prophylaxis against early anaphylactic reactions to antivenom for bothrops snakebites. BMJ. 1999;318:1451-1452.

15. Dassanayake AS, Karunanayake P, Kasturiratne KT, et al. Safety of subcutaneous adrenaline as prophylaxis against acute adverse reactions to anti-venom serum in snakebite. Ceylon Med J. 2000;47:48-49.

16. Premawardhena AP, de Silva CE, Fonseka MM, Gunatilake SB, de Silva HJ. Low dose subcutaneous adrenaline to prevent acute adverse reactions to antivenom serum in people bitten by snakes: randomised, placebo controlled trial. BMJ. 1999;318:1041-1043.

17. Gawarammana IB, Kularatne SA, Dissanayake WP, Kumarasiri RP Senanayake N, Ariyasena H. Parallel infusion of hydrocortisone \pm chlorpheniramine bolus injection to prevent acute adverse reactions to antivenom for snakebites. Med J Aust. 2004;180:20-23.

18. de Silva HA, Pathmeswaran A, Jayamanne S, et al. Prevention of acute adverse reactions to snake antivenom after snakebite: multi-centre, randomized, controlled clinical trial. PLoS Med. 2011;8:e1000435. 
19. Habib AG. Effect of pre-medication on early adverse reactions following antivenom use in snake bite: a systematic review and meta-analysis. Drug Saf. 2011;34:869-880

20. Rusznak C, Peebles RS. Anaphylaxis and anaphylactoid reactions: a guide to prevention, recognition and emergent treatment. Postgrad Med. 2002;111: 101-104.
21. Caron EJ, Manock SR, Maudin J, et al. Apparent marked reduction in early antivenom reactions compared to historical controls: was it prophylaxis or method of administration? Toxicon. 2009;54:779-783.

22. Williams DJ, Jensen SD, Nimorakiotakis B, Muller R, Winkel KD. Antivenom use, premedication and early adverse reactions in the management of snakebites in rural Papua New Guinea. Toxicon. 2007;49:780-792. 